\title{
A small cosmological constant from Abelian symmetry breaking
}

\author{
Gianmassimo Tasinato \\ Institute of Cosmology \& Gravitation, \\ University of Portsmouth, \\ Dennis Sciama Building, Portsmouth, \\ PO1 3FX, United Kingdom
}

(Dated: April 2014)

\begin{abstract}
We investigate some cosmological consequences of a vector-tensor theory where an Abelian symmetry in the vector sector is slightly broken by a mass term and by ghost-free derivative self-interactions. When studying cosmological expansion in the presence of large bare cosmological constant $\Lambda_{c c}$, we find that the theory admits branches of de Sitter solutions in which the scale of the Hubble parameter is inversely proportional to a power of $\Lambda_{c c}$. Hence, a large value of $\Lambda_{c c}$ leads to a small size for the Hubble scale. In an appropriate limit, in which the symmetry breaking parameters are small, the theory recovers the Abelian symmetry plus an additional Galileon symmetry acting on the longitudinal vector polarization. The approximate Galileon symmetry can make the structure of this theory stable at the energy scales we are interested in. We also analyze the dynamics of linearized cosmological fluctuations around the de Sitter solutions, showing that no manifest instabilities arise, and that the transverse vector polarizations become massless around these configurations.
\end{abstract}

\section{INTRODUCTION}

One of the main motivations for considering modifications of Einstein's general relativity (GR) is to understand why the universe is accelerating today. Cosmological acceleration might be due to the dynamics of gravity itself, that at very large distances deviates from the predictions of GR in such a way to provide accelerated expansion with no need of energy momentum tensor. This phenomenon is dubbed self-acceleration. The DGP model [1] is among the most studied examples, but various other proposals have been explored in the literature. Sometimes, the self-accelerating branches of cosmological solutions in these set-ups are plagued by instabilities. Nevertheless, the exploration of modified gravity models is certainly worthwhile for better understanding subtleties associated with the dynamics of gravity, and for suggesting new theoretical ideas to address the dark energy problem. See [2] for a comprehensive review on this subject.

Yet, most of these theories do not attempt to solve the classic cosmological constant problem, since they do not try to explain why the bare vacuum energy does not curve the space-time. In absence of symmetries, one would expect that quantum effects give contributions to vacuum energy that scale as the fourth power of the cut-off of the theory. In the theory of general relativity, without imposing additional symmetries beyond diffeomorphism invariance, the natural cut-off is the Planck mass. Hence, the expected size of the cosmological constant would scale as Planck mass to the fourth, that is 120 orders of magnitude larger than the measured dark energy scale today. See [3] for a classic review on the cosmological constant problem, and [4, 5] for recent perspectives on this topic. The work [3] also contains a powerful result, the so called Weinberg no-go theorem, 
that seems to forbid adjustment mechanisms for reducing the size of the cosmological constant; see however [6 11] for some interesting recent proposals for avoiding Weinberg's arguments.

The cosmological constant problem might be due to the particular structure and symmetries of GR. Enriching the theory with new symmetries, as for example supersymmetry or conformal symmetry, might help to reduce the size of the problem by many orders of magnitude. On the other hand, at least within the energy scales probed by current laboratory experiments, these additional symmetries are not manifest, hence they cannot be effective for reducing to acceptable levels the size of the cosmological constant.

In this work, we point out that a vector field with an approximate Abelian symmetry might shed some light on why the cosmological constant does not curve the space. We elaborate on the theory presented in [12], in which the Abelian symmetry of a vector coupled to gravity is broken by a mass term and derivative self-interactions. (See also [13] for a proposal to describe Dark Energy by means of a theory breaking an Abelian symmetry.) The theory is ghost-free and propagates three degrees of freedom in the vector sector: the two transverse vector components and a longitudinal scalar field. In an appropriate limit, in which the symmetry breaking parameters are small, the theory recovers an Abelian symmetry plus a Galileon symmetry acting on the longitudinal scalar mode. We study the behavior of cosmological expansion in this set-up in the presence of a large bare cosmological constant $\Lambda_{c c}$. We show that, interestingly, there are branches of de Sitter cosmological solutions in which the square of the Hubble parameter is inversely proportional to a power of $\Lambda_{c c}$. Hence, the larger the bare cosmological constant is, the smaller the value of the Hubble constant, and parameters can be chosen in such a way that the resulting Hubble scale is of the order of the observed dark energy scale. The existence of these cosmological solutions depends on the particular structure of our vector theory: exploiting approximate Galileon symmetries in the longitudinal sector, we argue that this structure is stable at the energy scales we are interested in. We also analyze the dynamics of cosmological fluctuations around these de Sitter solutions, showing that no manifest instabilities arise.

For simplicity, in this work we will not discuss the role of standard matter in cosmological expansion, and focus on the dynamics of the vector. Also, we will not be specific on the nature of the vector we consider. It could be the observed photon; or, alternatively, it could be another light vector field not belonging to the Standard Model. Contrarily to other hypothetical symmetries, an Abelian gauge symmetry acting on the photon field seems to be a fundamental symmetry realized in nature, at least to a level that did not manifest any appreciable violation so far: see [14] for an excellent review on photon mass limits. Yet, the study the cosmological consequences of tiny violation of Abelian gauge symmetries is interesting, since being associated with a long-range force might have an important role for shaping the structure of our universe at very large scales.

Various vector tensor theories have been developed so far with interesting cosmological applications. The first ones are [15, 16], while more recent well studied scenarios are the Einstein- Aether theory [17] and the TeVeS covariantized version of MOND [18]. For more recent examples see e.g. [19], and more generally see [2] for a comprehensive review on the subject with a complete list of references to the relevant literature. The specific feature of our approach is the emphasis on symmetry arguments for discussing our theory, in particular the explicit connections with Galileons. This fact can allow us to keep the structure of our set-up under control within the cosmological scales we are interested in.

The work is organized as follows. In Section 11 we present the set-up under consideration, determining homogeneous cosmological solutions. In Section III we study the dynamics of cosmological perturbations around these solutions. In Section IV we apply our findings to analyze the size of the dark energy scale, showing that there are interesting branches of solutions in which the Hubble scale is inversely proportional to the bare cosmological constant. We conclude in Section V. 


\section{THE SYSTEM UNDER CONSIDERATION}

We consider a theory of vector fields coupled to gravity, described by an action

$$
\mathcal{S}=\int d^{4} x \sqrt{-g}\left[\frac{M_{*}^{2}}{2} R-\frac{1}{4} F_{\mu \nu} F^{\mu \nu}-\mathcal{L}_{(0)}^{c o v}-\mathcal{L}_{(1)}^{c o v}-\mathcal{L}_{(2)}^{c o v}-\Lambda_{c c}\right]
$$

where $M_{*}^{2} R / 2$ is the Einstein-Hilbert term weighted by the square of Planck scale, $-F_{\mu \nu} F^{\mu \nu} / 4$ is the standard kinetic term for a vector potential $A_{\mu}, \Lambda_{c c}$ is a bare cosmological constant, and the vector interactions $\mathcal{L}_{(i)}^{\text {cov }}$ that break the Abelian symmetry are defined as

$$
\begin{aligned}
\mathcal{L}_{(0)}^{\text {cov }} & =m^{2} A_{\mu} A^{\mu}, \\
\mathcal{L}_{(1)}^{\text {cov }} & =\beta_{1} A_{\mu} A^{\mu}\left(\nabla_{\rho} A^{\rho}\right), \\
\mathcal{L}_{(2)}^{\text {cov }} & =\frac{\beta_{2}}{m^{2}} A_{\mu} A^{\mu}\left[\left(\nabla_{\rho} A^{\rho}\right)\left(\nabla_{\nu} A^{\nu}\right)-\left(\nabla_{\rho} A^{\nu}\right)\left(\nabla^{\rho} A_{\nu}\right)-\frac{1}{4} R A_{\sigma} A^{\sigma}\right] .
\end{aligned}
$$

This is the minimal ghost-free Lagrangian studied in [12] that couples a vector field with gravity, and leads to cosmological solutions with interesting features that we are going to analyze. $\mathcal{L}_{(0)}^{\text {cov }}$ is a Proca mass term, while $\mathcal{L}_{(1,2)}^{c o v}$ are ghost-free derivative self-interactions. The structure of the selfinteractions is chosen in such a way that the equation of motion for the time-component $A_{0}$ of the vector field does not contain time derivatives. Hence the equation for $A_{0}$ is a constraint equation that fixes one degree of freedom, and the theory propagates only three degrees of freedom in the vector sector ${ }^{1}$. The Lagrangian can be further generalized maintaining the ghost free condition, see for example [21]: however the minimal form of the action that we consider is particularly interesting for us because of symmetry properties that we will exploit in what follows. We add a bare cosmological constant $\Lambda_{c c}$ in order to analyze how cosmological solutions depend on its size, but for simplicity we will not explicitly discuss couplings with standard matter - although we will comment on this topic from time to time.

In general, this theory does not have any symmetry besides the diffeomorphism invariance of GR: indeed, the Lagrangians $\mathcal{L}_{(i)}^{c o v}$ break the $U(1)$ Abelian gauge symmetry $A_{\mu} \rightarrow A_{\mu}+\partial_{\mu} \xi$. On the other hand, as explained in [12], there exists a limit in which, by neglecting gravity and taking small values for $m, \beta_{i}$ the theory acquires Abelian and Galileon symmetries acting on the transverse and longitudinal vector polarizations. This limit is made particularly manifest by adopting a Stückelberg approach, and supplementing the Lagrangians $\mathcal{L}_{(i)}^{c o v}$ of eqs (2) 4) with interactions to a new scalar $\pi$, introduced in such a way to obtain a gauge-symmetric theory

$$
\begin{aligned}
\mathcal{L}_{(0)}^{\text {cov }}= & m^{2}\left(A_{\mu}+\frac{1}{\sqrt{2} m} \partial_{\mu} \pi\right)\left(A^{\mu}+\frac{1}{\sqrt{2} m} \partial^{\mu} \pi\right) \\
\mathcal{L}_{(1)}^{\text {cov }}= & \beta_{1}\left(A_{\mu}+\frac{1}{\sqrt{2} m} \partial_{\mu} \pi\right)\left(A^{\mu}+\frac{1}{\sqrt{2} m} \partial^{\mu} \pi\right)\left(\nabla_{\rho} A^{\rho}+\frac{1}{\sqrt{2} m} \square \pi\right), \\
\mathcal{L}_{(2)}^{\text {cov }}= & \frac{\beta_{2}}{m^{2}}\left(A_{\mu}+\frac{1}{\sqrt{2} m} \partial_{\mu} \pi\right)\left(A^{\mu}+\frac{1}{\sqrt{2} m} \partial^{\mu} \pi\right) \\
\times & {\left[\left(\nabla_{\rho} A^{\rho}+\frac{\square \pi}{\sqrt{2} m}\right)\left(\nabla_{\nu} A^{\nu}+\frac{\square \pi}{\sqrt{2} m}\right)-\left(\nabla_{\rho} A^{\nu}+\frac{\nabla_{\rho} \partial^{\nu} \pi}{\sqrt{2} m}\right)\left(\nabla_{\nu} A^{\rho}+\frac{\nabla_{\nu} \partial^{\rho} \pi}{\sqrt{2} m}\right)\right.} \\
& \left.\quad-\frac{1}{4} R\left(A_{\sigma}+\frac{1}{\sqrt{2} m} \partial_{\sigma} \pi\right)\left(A^{\sigma}+\frac{1}{\sqrt{2} m} \partial^{\sigma} \pi\right)\right] .
\end{aligned}
$$

\footnotetext{
${ }^{1}$ A similar set of interactions is studied in [20], but the coupling of the Ricci scalar with gravity is not taken in due account, rendering the theory not ghost-free when dynamical gravity is considered.
} 
The field $\pi$ plays the same physical role of the longitudinal vector mode in the original theory; on the other hand, now the interactions listed above preserve the Abelian gauge symmetry $A_{\mu} \rightarrow$ $A_{\mu}+\partial_{\mu} \xi$ at the price of introducing the degree of freedom $\pi$ that simultaneuosly transforms as $\pi \rightarrow \pi-\sqrt{2} m \xi$.

Let us now consider the following limit that decouples the dynamics of transverse and longitudinal vector modes:

$$
m \rightarrow 0 \quad, \quad \beta_{1} \rightarrow 0 \quad, \quad \beta_{2} \rightarrow 0 .
$$

This limit must be taken in a special way: indeed we require the following conditions for the ratios among the previous parameters in the limit in which they go to zero

$$
\frac{\beta_{1}}{m^{3}} \equiv \frac{\hat{\beta}_{1}}{\Delta^{3}}=\text { fixed } \quad, \quad \frac{\beta_{2}}{m^{6}} \equiv \frac{\hat{\beta}_{2}}{\Delta^{6}}=\text { fixed }
$$

In the previous expression, $\hat{\beta}_{1,2}$ are dimensionless constants, while $\Delta$ is a quantity of dimension of a mass. In this decoupling limit, when neglecting gravity, the theory enjoys an Abelian symmetry acting on the vector sector only $A_{\mu} \rightarrow A_{\mu}+\partial_{\mu} \xi$, and (as we will see in a moment) a Galileon symmetry $\pi \rightarrow \pi+c+b_{\mu} x^{\mu}$ for the scalar sector $\pi$, with a strong coupling Galileon scale set by $\Delta .{ }^{2}$ The fact that we recover symmetries in the limit in which $m, \beta_{1}$ and $\beta_{2}$ are going to zero implies that choosing these parameters small is technically natural in the 't Hooft sense [23]: at the quantum level, we do not expect large contributions to their size proportional to powers of the cut-off, but only corrections proportional to the size of the small parameters themselves.

Let us return to discuss the sector of the theory associated with the longitudinal vector polarization. In the decoupling limit (8), (9), the effective Lagrangian density for the vector longitudinal polarization $\pi$ reads

$$
\mathcal{L}_{\pi}^{\text {dec }}=-\frac{1}{2}(\partial \pi)^{2}+\frac{\hat{\beta}_{1}}{\Delta^{3}} \mathcal{L}_{(3)}^{\text {gal }}+\frac{\hat{\beta}_{2}}{\Delta^{6}} \mathcal{L}_{(4)}^{\text {gal }}
$$

where $\mathcal{L}_{(3,4)}^{\text {gal }}$ are the cubic and quartic Galileon Lagrangians discussed in [22]; see [12] for more details. In this limit we can exploit the non-renormalization theorems of Galileon interactions [22, 24, 25]. Our choice of operators in (1), although special, is technically natural since quantum corrections do not spoil the structure of the theory when the vev of $\pi$ is of order $\Delta$. On the other hand, the coupling with gravity breaks the Galileon symmetry (while it preserves the Abelian vector symmetry, and by construction maintains equations of motion of second order). In a cosmological setting, corrections associated with gravitational effects are expected to be small in the limit in which the strong coupling scale $\Delta$ is larger than the Hubble scale of the space-time under consideration, $H \ll \Delta[26,27]$. At the same time, Planck suppressed operators induced by quantum corrections are expected to be negligible in the limit in which the strong coupling scale is much smaller than the gravitational cut-off, $\Delta \ll M_{\star}$. Further corrections to the structure of the Lagrangians contained in eq. (11) can arise since finite values for the vector mass and self-interaction coefficients $m, \beta_{i}$ break the Galileon symmetry by coupling longitudinal and transverse degree of freedom - on the other hand as argued above these couplings can be sufficiently suppressed by choosing (technically natural) small values for these parameters. Finally, additional corrections can arise when coupling the theory to standard matter - unless this is done in a careful way such to maintain the above symmetries to a certain extent. This is an important subject, since couplings

\footnotetext{
${ }^{2}$ At least if the $\hat{\beta}_{i}$ are of order one: if these parameters are small, the strong coupling scale can be higher.
} 
with matter will also govern the scale of the typical vev for the vector longitudinal polarization $\pi$, offering the possibility to develop screening effects analogous to the gravitational Vainshtein mechanism [28] (see also [29] for a realization of a Vainshtein mechanism in a vector-scalar-tensor theory of gravity). This subject has been partially explored in [12], but will be further developed elsewhere.

These considerations suggest that, when limiting our interest to cosmological space-times with Hubble parameter smaller than the strong coupling scale $\Delta$ for the vector longitudinal interactions, it is possible to maintain a sufficient degree of symmetry to protect in a technically natural way the structure of our theory. This is a feature that will have important implications for our arguments. Let us then pass to analyze, for the remaining of this section, the homogenous cosmological evolution associated with the above theory, extending results first presented in [12]. The Ansätze for the FRW background metric and vector profiles that we consider are

$$
\begin{aligned}
d s^{2} & =-d t^{2}+a^{2}(t) \delta_{i j} d x^{i} d x^{j} \\
A_{\mu} & =\left(A_{0}(t), 0,0,0\right)
\end{aligned}
$$

Notice that the homogeneous vector profile does not break spatial isotropy. The homogenous equation of motion for the vector component is a constraint equation, that reads

$$
A_{0}\left(m^{4}-3 \beta_{1} m^{2} A_{0} H+9 \beta_{2} A_{0}^{2} H^{2}\right)=0,
$$

where $H=\dot{a} / a$ is the Hubble parameter. The above algebraic equation, a part from the solution $A_{0}=0$ (that does not lead to interesting cosmological expansion), admits the solutions

$$
\begin{aligned}
& A_{0}=\frac{c_{ \pm} m^{2}}{H}, \\
& c_{ \pm}=\frac{\beta_{1} \pm \sqrt{\beta_{1}^{2}-4 \beta_{2}}}{6 \beta_{2}},
\end{aligned}
$$

where from now on we set $m^{2}>0, \beta_{1}>0$. Hence we learn that, in a homogeneous FRW setting, the constraint equation for $A_{0}$ leads to a profile for this field that is inversely proportional to the Hubble parameter. In order to ensure real values for $c_{ \pm}$, we will impose $\beta_{2} \leq \beta_{1}^{2} / 4$. For handling more easily our formulae, it is convenient to make the following parameter redefinition:

$$
\begin{aligned}
\beta_{2} & =\frac{\left(1-\gamma^{2}\right) \beta_{1}^{2}}{4}, \\
\Lambda_{c c} & =\frac{m^{3} M_{*}}{3 \beta_{1}} \lambda,
\end{aligned}
$$

where $\gamma, \lambda$ are dimensionless quantities. This implies that we trade $\beta_{2}$ for $\gamma$; in terms of the parameters $\hat{\beta}_{i}$ of eq. (9) (useful to investigate the decoupling limit (8) in which we recover Abelian and Galileon symmetries) we can write

$$
\hat{\beta}_{2}=\frac{\left(1-\gamma^{2}\right) \hat{\beta}_{1}^{2}}{4} .
$$

That is, $\gamma$ is not affected by the limit (8). Notice that eq (17) means that we are going to use the dimensionless parameter $\lambda$ to quantify the size of the cosmological constant. For the moment we do not impose any requirement on the size of $\lambda$, that could also be very large (so to drive $\Lambda_{c c}$ up to the gravitational cut-off of our theory). How the cosmological expansions depends on $\lambda$ and then 
on the bare cosmological constant $\Lambda_{c c}$ will be the main topic of Section IV. Using eq. (16), the parameter $c_{ \pm}$reads

$$
c_{ \pm}=\frac{2}{3 \beta_{1}(1 \mp|\gamma|)} .
$$

Notice that the $c_{ \pm}$are distinguished by the sign in front of $|\gamma|$. Hence in what follows, without lack of generality, we will write in an unified way these two branches as

$$
c_{0}=\frac{2}{3 \beta_{1}(1+\gamma)},
$$

and allow for an arbitrary sign of $\gamma$. In terms of these parameters, the Einstein equations reduce to the following condition for the Hubble parameter

$$
0=H^{2}\left(-2 \Lambda_{c c}+6 H^{2} M_{\star}^{2}-2 m^{2} A_{0}^{2}+12 \beta_{1} H A_{0}^{3}-\frac{45 \beta_{2}}{m^{2}} H^{2} A_{0}^{4}\right)
$$

and substituting (14) in the previous equation we find two allowed branches of non-vanishing constant solutions for the Hubble parameter

$$
H_{\mp}^{2}=\frac{m^{3}}{18 \beta_{1} M_{*}}\left[\lambda \mp \sqrt{\lambda^{2}-\frac{24(1+3 \gamma)}{(1+\gamma)^{3}}}\right] .
$$

So, we learn that the higher order self-couplings of the vector, controlled by the interaction Lagrangians (24 (4), switch on a non-trivial time-dependent profile for the component $A_{0}(t)$, that drives cosmological expansion. Choosing parameters such that the right hand side of eq. (22) is positive, the resulting cosmological evolution corresponds to a de Sitter universe with constant Hubble rate. To have a positive argument for the square root in (22), we impose the following condition on the dimensionless parameters $\lambda$ and $\gamma$ :

$$
\lambda^{2} \geq \frac{24(1+3 \gamma)}{(1+\gamma)^{3}}
$$

After imposing (23), we can distinguish two options to obtain a positive value for the square of the Hubble parameter:

1. If $\lambda$ is positive, the positive branch $H_{+}$is always well defined, in the sense that $H_{+}^{2}$ is positive. In the case of negative branch $H_{-}$, moreover, to have a positive $H_{-}^{2}$ we have to additionally demand

$$
\frac{1+3 \gamma}{(1+\gamma)^{3}} \geq 0 \quad \rightarrow \quad \gamma \leq-1 \text { or } \gamma \geq-\frac{1}{3}
$$

2. If $\lambda$ is negative, the negative branch $H_{-}$is never well defined. In the case of positive branch $H_{+}$, moreover, to have a positive $H_{+}^{2}$ we have to additionally demand

$$
\frac{1+3 \gamma}{(1+\gamma)^{3}} \leq 0 \quad \rightarrow \quad-1 \leq \gamma \leq-\frac{1}{3}
$$

The negative branch $H_{-}$for the Hubble parameter in eq (22) appears particularly interesting, since the minus sign inside the square parenthesis in (22) compensates possibly large contributions associated with the parameter $\lambda$ (appearing in the expression (17) for the bare cosmological constant). We will explore in detail this feature in section IV] In the next section, instead, we analyze the behavior of cosmological fluctuations around the homogeneous configurations we have determined. 


\section{DYNAMICS OF LINEARIZED PERTURBATIONS}

The dynamics of cosmological fluctuations around the previous background solutions can be analyzed straightforwardly. It is convenient to split perturbations into tensor, vector, and scalar components with respect to the spatial sections of the FRW background geometry, and implement an ADM approach. Let us start counting the available degrees of freedom (dofs). We start with ten dofs in our metric, plus four dofs for the vector. The theory respects diffeomorphism invariance of GR, that removes four dofs. Moreover, we still have the four constraints of GR, plus one constraint of the vector action (associated with the equation of motion for $A_{0}[12]$ ): these constraints remove five dofs. In total, we remain with five dofs: two transverse traceless tensor components, two transverse vector components, one scalar component.

Using diffeomorphism invariance, a gauge can be selected for metric fluctuations such that we can write

$$
g_{\mu \nu}=-(1+N)^{2} d t^{2}+a^{2}(t)\left(e^{2 \zeta} \delta_{i j}+h_{i j}\right)\left(d x^{i}+N^{i} d t\right)\left(d x^{j}+N^{j} d t\right),
$$

where $N, N_{i}, \zeta, h_{i j}$ are small fluctuations around the background FRW solution characterized by a scale factor $a(t) . N$ and $N_{i}$ are the lapse and shift perturbations, whose linearized equations of motion provide the the Hamiltonian and momentum constraints of GR. $\zeta$ is a scalar fluctuation, while $h_{i j}$ is a tensor fluctuation satisfying a transverse-traceless condition $h_{i i}=0, \partial_{i} h_{i j}=0$. Vector fluctuations in the metric are set to zero by gauge choice. These conditions do not completely fix the gauge: at zero momentum we have the additional freedom to shift the time coordinate $t \rightarrow t+\epsilon(t)$ and rescale the spatial coordinates $x^{i} \rightarrow(1+q) x^{i}$ by small quantities [30].

For what respect the vector sector with slightly broken Abelian symmetry, it is convenient to use the Stückelberg approach, and write $A_{\mu}$ as

$$
A_{\mu}=\left(A_{0}+\dot{\pi}, \hat{A}_{i}^{T}+\partial_{i} \pi\right) .
$$

$A_{0}$ corresponds to the homogeneous background solution discussed in the previous section, while $\hat{A}_{i}^{T}$ and $\pi$ play the role of transverse vector and scalar fluctuations. We introduced a Stuckelberg field $\pi$ restoring the Abelian invariance, that we use to choose an Abelian gauge where the fluctuation $\hat{A}_{0}=0$. Notice that in this description we apparently have six dofs, instead of the expected five, so one of the scalars is actually a gauge mode. Since in our system gravity is non-minimally coupled with the vector Lagrangian, we expect that the dynamical scalar dof will be a mixture between the scalars $\zeta$ and $\pi$. It is convenient to work with both these scalars to start with, and leave the constraint equations to determine the scalar combination playing a physical role in what follows.

\section{A. Tensor perturbations}

The quadratic action for tensor perturbations can be found straightforwardly. Notice that, when $\beta_{2}$ is non-vanishing (i.e. $|\gamma| \neq 1$, see eq (16) ) the vector is non-minimally coupled with the metric: hence the gauge field background value $A_{0}$ 'renormalizes' the Planck mass. Indeed, one finds the following effective Lagrangian density at quadratic level for the tensor fluctuations $h_{i j}$

$$
\mathcal{L}_{\text {tens }}^{(\text {quad })}=\frac{M_{ \pm}^{2}}{2} \mathcal{L}_{E H}^{(\text {quad })},
$$


where $\mathcal{L}_{E H}^{(\text {quad })}$ is the expansion of the Einstein-Hilbert action at quadratic order, and the effective Planck scale is given by

$$
M_{ \pm}^{2}=M_{*}^{2}\left(1-\frac{3 \beta_{2} c_{0}^{4} m^{6}}{2 H_{ \pm}^{4} M_{*}^{2}}\right) .
$$

In the previous formula, the \pm denotes the choice of branch of background solutions for the Hubble parameter in eq. (22). Using the results of Section II $M_{ \pm}$can be expressed as

$$
M_{ \pm}^{2}=\left(1+\frac{24(1+\gamma)}{(\gamma-1)^{3}\left(\lambda \pm \sqrt{\lambda^{2}-\frac{24(3 \gamma-1)}{(\gamma-1)^{3}}}\right)^{2}}\right) M_{*}^{2}
$$

In order to have a consistent set-up, we impose $M_{ \pm}^{2}>0$. Hence, if $\gamma \neq-1$ the effective Planck scale depends on the value of the cosmological constant, since it explicitly depends on $\lambda$, the parameter that controls $\Lambda_{c c}$ (see eq (17)). Let us point out that the quantity $M_{ \pm}^{2}$ of eq (30) can be interpreted as parameterizing the self-coupling scale of gravitational interactions. On the other hand, if vector fields directly couple with standard matter, they can also have a role in determining the effective coupling of gravity with any additional matter content.

\section{B. Vector perturbations}

Also vector fluctuations are not difficult to deal with. By splitting the metric shift vector $N_{i}=N_{i}^{T}+\partial_{i} \psi$, with $N_{i}^{T}$ the transverse components and $\psi$ the longitudinal part, the momentum constraint imposes $N_{i}^{T}=0$. Interestingly, a straightforward calculation shows that the mass of the transverse vector fluctuations $\hat{A}_{i}^{T}$ exactly vanishes around the background cosmological configurations we are considering: at quadratic order, the Lagrangian density for the vector fluctuations $\hat{A}_{i}^{T}$ only contains the standard kinetic terms:

$$
\mathcal{L}_{\text {vec }}^{(\text {quad })}=-\frac{1}{4} F_{\mu \nu} F^{\mu \nu}
$$

Hence, we are dealing with a transverse vector fluctuations with healthy kinetic terms and zero mass (although the transverse polarizations acquire interactions with the longitudinal component at third order in perturbations). If we interpret the vector we are dealing with as the photon, this implies that the usual constraints on the photon mass do not directly apply in the present context, since the vector is massless. It would be interesting to study in detail the phenomenological consequences of the higher order interactions associated with Lagrangians $\mathcal{L}_{(1)}^{\text {cov }}$ and $\mathcal{L}_{(2)}^{\text {cov }}$, that can lead to screening mechanisms analogous to the gravitational Vainshtein mechanism. This will be the subject of a future work.

\section{Scalar perturbations}

The analysis of scalar vector fluctuations is also straightforward. The Hamiltonian and momentum constraint equations, using also the gauge freedom left at zero momentum, provide the following conditions (recall that $\psi$ is the longitudinal scalar part of the shift perturbations 
$\left.N_{i}=N_{i}^{T}+\partial_{i} \psi\right)$

$$
\begin{aligned}
N & =\frac{H_{ \pm}}{c_{0} m^{2}} \dot{\pi}, \\
\psi & =-\frac{H_{ \pm}}{c_{0} m^{2}} \pi, \\
\zeta & =\frac{H_{ \pm}^{2}}{c_{0} m^{2}} \pi .
\end{aligned}
$$

So all the quantities can be expressed in terms of the Stückelberg scalar $\pi$, physically corresponding to the vector longitudinal polarization, and as expected one ends with a single (potentially) dynamical scalar fluctuation. However, by imposing the above constraints to the action of quadratic scalar fluctuations around the homogeneous background, one ends with a total derivative signaling a trivial dynamics for the scalar $\pi$ :

$$
\mathcal{L}_{\text {scal }}^{(\text {quad })}=0
$$

This implies that at quadratic level our action propagates only four dofs around the homogeneous de Sitter background, in the tensor and vector sectors. It remains to be checked whether higher order contributions in the fluctuations induce instabilities in the scalar sector, analogously to what happens, for example, in the vector sector of massive gravity [31, 32]. On the other hand, let us point out that this problem, if it really exists, might be cured by suitable couplings with matter sector, that might be able to induce healthy kinetic terms for $\pi$. We postpone a detailed analysis of this issue for future work, and we pass to discuss the consequences of our findings so far for the dark energy scale.

\section{THE DARK ENERGY SCALE}

We have seen that coupling our vector Lagrangian with gravity we are able to find cosmological solutions corresponding to a de Sitter universe. We include the effect of a bare cosmological constant $\Lambda_{c c}$, that in eq (17) we parameterized in terms of the available parameters as

$$
\Lambda_{c c}=\frac{m^{3} M_{*}}{3 \beta_{1}} \lambda \text {. }
$$

In the previous expression, $\lambda$ is a dimensionless parameter, whose value could also be large so to push the size of $\Lambda_{c c}$ towards the gravitational cut-off of the theory. In the remaining of this section, for definiteness, we will focus on the case in which $\lambda$ is positive. The homogeneous cosmological configuration is characterized by a de Sitter expansion: there are two available branches for the Hubble parameter (see eq. (22)

$$
H_{\mp}^{2}=\frac{m^{3}}{18 \beta_{1} M_{*}}\left[\lambda \mp \sqrt{\lambda^{2}-\frac{24(1+3 \gamma)}{(1+\gamma)^{3}}}\right] .
$$

In discussing fluctuations, we have learned that scalar fluctuations do not propagate at quadratic level around this de Sitter space. Vector fluctuations propagate as massless modes, while tensor fluctuations are described by a quadratic expansion of the Einstein Hilbert action, with an induced renormalization of the effective Planck scale (see eqs (29) and (30)):

$$
M_{\mp}^{2}=\left(1-\frac{24(1-\gamma)}{(1+\gamma)^{3}\left(\lambda \mp \sqrt{\lambda^{2}-\frac{24(1+3 \gamma)}{(1+\gamma)^{3}}}\right)^{2}}\right) M_{*}^{2} .
$$


In the previous formula, a plus or minus sign depends on the choice for the branch of the Hubble parameter in eq (37). $M_{\mp}$ correspond to the effective Planck mass in the de Sitter solution of interest, while $M_{\star}$ is the original gravitational Planck scale around a flat solution with no homogeneous vector profile.

\section{A. Large bare cosmological constant}

These results have potentially interesting consequences for the scale of dark energy. Let us start by considering the case in which the numerical coefficient $\lambda$ in eq (36) is positive and large (with 'large' we mean $\lambda$ much bigger than $(1+3 \gamma) /(1+\gamma))$ corresponding to the case of potentially large vacuum energy $\left|\Lambda_{c c}\right|$.

We obtain for the two branches (37) of the Hubble parameter the following limiting values

$$
\begin{aligned}
& H_{-}^{2} \simeq \frac{2(1+3 \gamma) m^{3}}{3 \beta_{1}(1+\gamma)^{3} \lambda M_{*}}, \\
& H_{+}^{2} \simeq \frac{\lambda m^{3}}{9 \beta_{1} M_{*}} .
\end{aligned}
$$

In this large $\lambda$ limit, the effective Planck mass for the two branches of solutions is

$$
\begin{aligned}
& M_{-}^{2} \simeq \frac{(1+\gamma)^{3}(\gamma-1) \lambda^{2}}{6(1+3 \gamma)^{2}} M_{*}^{2}+\frac{(3+\gamma)}{(1+3 \gamma)} M_{*}^{2}, \\
& M_{+}^{2} \simeq M_{*}^{2} .
\end{aligned}
$$

The simplest and most interesting case to analyze corresponds to the negative branch, $H_{-}, M_{-}$, and $\gamma=1$. In this case, the parameter $\beta_{2}=0$, and eq (41) tells us that the Planck mass does not get renormalized, $M_{-}=M_{\star}$. Eq (39) leads to

$$
\begin{aligned}
H_{-}^{2} & =\frac{m^{3}}{3 \beta_{1} \lambda M_{\star}} \\
& =\left(\frac{m^{3}}{3 \beta_{1}}\right)^{2} \frac{1}{\Lambda_{c c}} \\
& =\left(\frac{\Delta^{3}}{3 \hat{\beta}_{1}}\right)^{2} \frac{1}{\Lambda_{c c}}
\end{aligned}
$$

In the second line of the previous expression, eq (44), we used eq (36): interestingly, the Hubble scale results inversely proportional to the value of the bare cosmological constant. In the third line, we used the definition in (9), showing that the previous relation holds also in the limit of very small parameters $m, \beta_{1}$ in which we recover the Abelian and the (approximate) Galileon symmetries. So, the larger is the bare cosmological constant $\Lambda_{c c}$, the smaller is $H_{-}^{2}$ : the actual value of $H_{-}^{2}$ then depends on the strong coupling scale $\Delta$ controlling the Galileonic self-interactions of the longitudinal vector polarization. For example, requiring that $H_{-}$is of order of present day Hubble scale,

$$
H_{-} \sim 10^{-33} \mathrm{eV},
$$

one finds

$$
\Delta \simeq\left(3 \hat{\beta}_{1}\right)^{\frac{1}{3}}\left(\frac{\Lambda_{c c}}{M_{\star}^{4}}\right)^{\frac{1}{6}} 10^{7} \mathrm{eV} .
$$


If $\hat{\beta}_{1} \sim 1$, and at the same time $\Lambda_{c c}$ is pushed towards the gravitational cut-off, the resulting $\Delta$ is an intermediate scale between $H_{-}$and $M_{\star}$, a particularly interesting situation since in the limit of small $m, \beta_{i}$ one recovers approximate Abelian and Galilean symmetries that protect the structure of our theory: see our discussion in Section II. Different strong coupling scales $\Delta$, that might required by additional phenomenological considerations, can be obtained by changing the value of $\hat{\beta}_{1}$, or reducing the size of $\Lambda_{c c}$ by considering a set-up with supersymmetry broken well above the electroweak scale. Also, notice that choosing a smaller $\Delta$ one can obtain a value for the Hubble parameter induced by $\Lambda_{c c}$ much suppressed with respect to its present day value, so to remove by this mechanism the contribution of the bare cosmological constant, and then explain present day acceleration with some other option.

The energy density stored in the vector field within this cosmological de Sitter solution is of the size of the bare cosmological constant: $\rho_{A} \sim \Lambda_{c c}$. Indeed, in this branch of solutions the constraint equation for $A_{0}$ automatically adjusts the vector contribution to the energy momentum tensor, in such a way to compensate to a large extent the bare cosmological constant - up to the small leftover collected in the right hand side of (45). Analogously to the scalar-tensor theory of [6], we can avoid Weinberg's no-go theorem [3] because we are considering cosmological de Sitter configurations and a non-constant field profile, while Weinberg's arguments apply only to situations in which full Poincaré invariance is maintained. Notice however that the vev of the field $A_{0}$ itself can reach trans-Planckian values: using eqs (14) and (44) one finds $A_{0} \sim \sqrt{\Lambda_{c c}} / m$. This is a feature shared with the scenario [8]. It is not clear if it can constitute a problem though, since the field $A_{0}$ is non-dynamical in our set-up ${ }^{3}$.

Let us briefly recap our findings so far. We found that the structure of the tensor-vector action we started with admits a branch of de Sitter cosmological solutions in presence of a large bare cosmological constant $\Lambda_{c c}$, but with a value for the Hubble parameter that is inversely proportional to a power of $\Lambda_{c c}$. This result depends on the particular set of operators we consider in our vector action, that after solving for all the constraints leads to a specific equation for the Hubble parameter with the properties discussed above. The structure of our theory can be argued to be technically natural, at least for the scales of interest and in the limit of small symmetry breaking parameters. Hence, with the help of symmetry arguments this set-up might provide the opportunity to keep in a natural way the dark energy scale much smaller than the cut-off of our theory.

The very same arguments can be applied in the case in which $\beta_{2}$ is turned on, that is $\gamma \neq 1$. However, in this case one has to take extra care to the renormalization of the Planck scale, see eq. (38) that can push the effective gravitational cut-off, and the bare cosmological constant, at values much larger than $M_{\star}^{4}$, possibly destabilizing relations as (47): choosing a sufficient small (and technically natural) value for $\beta_{2}$, on the other hand, one can check that the previous arguments hold with little changes.

While until now we focussed on the interesting class of solutions corresponding to the negative branch $H_{-}$let us briefly explain what happens for the positive branch case. In the limit of large $\lambda$, the positive branch $H_{+}$can still drive acceleration, but in this case the value of the square of the Hubble parameter is directly proportional to the scale of the bare cosmological constant, see eq. (40). Hence this branch of solutions does not have much to say with respect to the cosmological constant problem, and is less interesting for the purpose of explaining present day acceleration.

\footnotetext{
${ }^{3}$ We thank Tony Padilla for discussions on these points.
} 


\section{B. Coupling with matter}

While until this stage we focussed on the situation of a universe filled with cosmological constant and vector fields, one can extend the analysis of the previous subsection to the case in which other degrees of freedom are included. Since our vector theory breaks the Abelian symmetry, nonstandard couplings between the vector sector and matter are allowed, that do not respect the gauge symmetry: precisely those couplings can be exploited for obtaining a standard cosmological evolution in our set-up. This is a subject that will be covered in full detail in 33]. Nevertheless let us start discussing this topic here, by discussing the easiest example of a massless scalar field $\phi$ with no self-interactions, but that couples with the vector. For simplicity, in this subsection we focus our attention to the case $\beta_{2}=0$, that as discussed at the beginning of section IVA has the advantage of not renormalizing the Planck mass. The kinetic term for the scalar Lagrangian is $\mathcal{L}_{m}(\phi, \partial \phi)=\frac{1}{2} \partial_{\mu} \phi \partial^{\mu} \phi$. At the homogeneous level, the energy density associated with such a kinetic term is $\rho=\dot{\phi}^{2} / 2$.

We allow for a direct, Planck suppressed coupling between the scalar and the vector field, and include the following contribution to the total action $\mathcal{S}$ of eq. (11)

$$
\mathcal{S}_{m}=\int d^{4} x \sqrt{-g}\left[-\frac{1}{2} \partial_{\mu} \phi \partial^{\mu} \phi+\frac{\xi}{2 M_{\star}^{2}} A_{\rho} A^{\rho} \partial_{\mu} \phi \partial^{\mu} \phi\right],
$$

where $\xi$ is a dimensionless coupling, whose value will be specified later. The new contribution (48) to the action renders the background solution for the vector dependent on $\rho$. Indeed, focussing again on configurations in which only the $A_{0}$ component is turned on, the vector equations of motion are solved by

$$
A_{0}=\frac{\left(m^{2} M_{\star}^{2}+\xi \rho\right)}{3 \beta_{1} M_{\star}^{2} H} .
$$

The Friedmann equation governing the evolution of the scale factor receives contributions from the scalar energy density $\rho$ and becomes

$$
H^{2}=\frac{\Lambda_{c c}}{3 M_{\star}^{2}}+\frac{\rho}{3 M_{\star}^{2}}+\frac{\xi A_{0}^{2} \rho}{3 M_{\star}^{4}}+\frac{m^{2} A_{0}^{2}}{3 M_{\star}^{2}}-2 \frac{\beta_{1} A_{0}^{3} H}{M_{\star}^{2}} .
$$

Plugging into the previous expression the profile (49) for $A_{0}$, and expanding for large values of the bare cosmological constant $\Lambda_{c c}$ (as we did in the previous section IVA), we end with the following expression for the Hubble parameter, that generalizes the 'negative branch' of solutions previously analyzed by including $\rho$ :

$$
H^{2}=\frac{m^{6}}{9 \beta_{1}^{2} \Lambda_{c c}}+\frac{\left(\xi m^{4} \Lambda_{c c}-m^{6} M_{\star}^{2}\right) \rho}{9 \beta_{1}^{2} M_{\star}^{2} \Lambda_{c c}^{2}}+\ldots
$$

where the ... contain subleading corrections. The first contribution to the right hand side is the constant term we found in eq. (45), inversely proportional to the bare cosmological constant. At this stage, it can be more conveniently expressed as

$$
\frac{m^{6}}{9 \beta_{1}^{2} \Lambda_{c c}} \equiv \frac{\bar{\Lambda}}{3 M_{\star}^{2}} \quad \rightarrow \quad m^{6}=\frac{3 \beta_{1} \Lambda_{c c} \bar{\Lambda}}{M_{\star}^{2}}
$$

where the quantity $\bar{\Lambda}$, in order not to curve excessively the space-time, has to satisfy the inequality $\bar{\Lambda} \leq\left(10^{-3} \mathrm{eV}\right)^{4} \simeq 10^{-120} M_{\star}^{4}$. These considerations are equivalent to the ones made around eqs. (46), (47). 
We can now choose the dimensionless parameter $\xi$ appearing in the second term of (51) such to obtain a standard form for the Friedmann equation. With this purpose we set

$$
\xi^{3}=\frac{3 \beta_{1}^{2} \Lambda_{c c} M_{\star}^{4}}{9 \bar{\Lambda}^{2}}\left(1+\frac{\bar{\Lambda}}{\Lambda_{c c}}\right)^{3} .
$$

Making this choice, one finds the familiar expression for the Friedmann equation

$$
H^{2}=\frac{\bar{\Lambda}}{3 M_{\star}^{2}}+\frac{\rho}{3 M_{\star}^{2}}+\ldots
$$

plus contributions that are subleading in the limit $\bar{\Lambda} \ll \Lambda_{c c}$. Hence we learn that by appropriately coupling matter with the vector field, as done in eq. (48) for this simple example of free scalar, one can reproduce standard cosmological evolution at the homogeneous level.

The actual size of the dimensionless parameter $\xi$ in eq. (53) can be chosen smaller than unity so to break mildly the Abelian symmetry. In order to achieve this condition, we have to take very small (but technically natural) values of $\beta_{1}$. Choosing a value for the bare $\Lambda_{c c}$ of the order of the gravitational cut-off scale, $\Lambda_{c c} \simeq M_{\star}^{4}$, and requiring (as explained above) $\bar{\Lambda} \leq 10^{-120} M_{\star}^{4}$, the requirement of having $\xi \leq 1$ leads to the inequality $\beta_{1} \leq 10^{-120}$. A very small number but - as argued in the previous sections - a technically natural quantity in the 't Hooft sense, since in the limit of small $m, \beta_{1}$ the theory recovers Abelian and Galileon symmetries.

\section{Small bare cosmological constant}

One can also be interested to what happens in the limit of small $\lambda, \Lambda_{c c}$, to understand to what extent our Lagrangian is able to drive cosmological acceleration exploiting the dynamics of the vector degrees of freedom. It turns out that it is not possible to completely switch off $\lambda$ and then $\Lambda_{c c}$. This since the stability of the tensor sector requires a positive effective Planck mass squared $M_{ \pm}^{2}$ for the two branches of solutions given eq. (38). When demanding that $M_{\star}^{2}>0$, this imposes the following condition for the two branches of solutions:

$$
\lambda\left(\lambda \mp \sqrt{\lambda^{2}-\frac{24(1+3 \gamma)}{(1+\gamma)^{3}}}\right) \geq \frac{24}{(1+\gamma)^{2}} .
$$

One can easily check that this inequality can be satisfied only if $|\gamma| \geq 1$. Imposing this condition implies that $(1+3 \gamma) /(1+\gamma)^{3}>0$, and in order to have a well definite square root in the previous formula we have to demand that a non-vanishing $\lambda$ is turned on, such to satisfy

$$
\lambda^{2} \geq \frac{24(1+3 \gamma)}{(1+\gamma)^{3}}
$$

Imposing the minimal value of $\lambda$ that satisfies such inequality, we find the following values for the physical parameters

$$
\begin{aligned}
& H_{ \pm}^{2}=\sqrt{\frac{2(1+3 \gamma)}{3(1+\gamma)^{3}}} \frac{m^{3}}{3 \beta_{1} M_{\star}}, \\
& M_{ \pm}^{2}=\frac{4 \gamma}{1+3 \gamma} M_{\star}^{2},
\end{aligned}
$$

and when expressing the value of the Hubble parameter in terms of $\Lambda_{c c}$ we find

$$
H_{ \pm}^{2}=\frac{2 \gamma}{3(1+3 \gamma)} \frac{\Lambda_{c c}}{M_{ \pm}^{2}}
$$


Hence in this case the vector field contributes to the accelerated expansion changing by a factor $2 \gamma /(1+3 \gamma)$ the value of the effective cosmological constant.

\section{CONCLUSIONS}

In this work, we studied cosmological consequences of the vector-tensor theory presented in [12], where the Abelian symmetry of a vector coupled to gravity is slightly broken by a mass term and ghost-free derivative self-interactions. When studying cosmological expansion in the presence of large bare cosmological constant $\Lambda_{c c}$, we found that the theory admits branches of de Sitter solutions in which the scale of the Hubble parameter is inversely proportional to a power of $\Lambda_{c c}$. Hence, the larger the bare cosmological constant is, the smaller the value of the Hubble constant, and parameters can be chosen in such a way that the resulting Hubble scale is of the order of the observed dark energy scale. The theory propagates up to three degrees of freedom in the vector sector: the two transverse vector components and a longitudinal scalar mode. In an appropriate limit, in which the symmetry breaking parameters are small, the theory recovers the Abelian symmetry plus an additional Galileon symmetry acting on the longitudinal scalar mode. The existence of the interesting branch of cosmological solutions depends on the particular structure of our vector theory: exploiting the approximate Galileon symmetries in the longitudinal sector, we argued that this structure can be stable for the energy scales we are interested in. We also analyzed the dynamics of cosmological fluctuations around the de Sitter solutions, showing that no manifest instabilities arise, and that the transverse vector polarizations become massless around these configurations.

We did not make explicit hypothesis on the nature of the vector field we considered: it could be the observed photon, or an additional light vector field not belonging to the Standard Model. The main task left to study is how normal matter gravitates in this cosmological set-up, and whether it couples with the vector field in a way that does not spoil the features of the cosmological solutions we studied. The longitudinal polarization of the vector field can mediate long-range forces, that can nevertheless be screened by an analogue of the Vainshtein mechanism. Normal matter, on the other hand, contributes to cosmological evolution both by directly curving the space-time, and by modifying the homogeneous time-dependent profile of the vector field when appropriate vector-matter couplings are included. A detailed study of these issues is left for future investigation.

\section{Acknowledgments}

It is a pleasure to thank Marco Crisostomi, Lavinia Heisenberg, Ivonne Zavala and especially Tony Padilla for comments on the draft, as well as STFC for financial support through the grant $\mathrm{ST} / \mathrm{H} 005498 / 1$.

[1] G. R. Dvali, G. Gabadadze and M. Porrati, "4-D gravity on a brane in 5-D Minkowski space," Phys. Lett. B 485 (2000) 208 hep-th/0005016.

[2] T. Clifton, P. G. Ferreira, A. Padilla and C. Skordis, "Modified Gravity and Cosmology," Phys. Rept. 513 (2012) 1 arXiv:1106.2476 [astro-ph.CO]].

[3] S. Weinberg, "The Cosmological Constant Problem," Rev. Mod. Phys. 61 (1989) 1.

[4] J. Polchinski, "The Cosmological Constant and the String Landscape," hep-th/0603249.

[5] C. P. Burgess, "The Cosmological Constant Problem: Why it's hard to get Dark Energy from Microphysics," arXiv:1309.4133 [hep-th]. 
[6] C. Charmousis, E. J. Copeland, A. Padilla and P. M. Saffin, "General second order scalar-tensor theory, self tuning, and the Fab Four," Phys. Rev. Lett. 108 (2012) 051101 [arXiv:1106.2000 [hep-th]].

[7] Y. Aghababaie, C. P. Burgess, S. L. Parameswaran and F. Quevedo, "Towards a naturally small cosmological constant from branes in 6-D supergravity," Nucl. Phys. B 680 (2004) 389 hep-th/0304256; C. P. Burgess, "Towards a natural theory of dark energy: Supersymmetric large extra dimensions," AIP Conf. Proc. 743 (2005) 417 hep-th/0411140; Y. Aghababaie et al., "Warped brane worlds in six-dimensional supergravity," JHEP 0309 (2003) 037 [hep-th/0308064].

[8] P. Batra, K. Hinterbichler, L. Hui and D. N. Kabat, "Pseudo-redundant vacuum energy," Phys. Rev. D 78 (2008) 043507 arXiv:0801.4526 [hep-th]].

[9] N. Kaloper and A. Padilla, "Sequestering the Standard Model Vacuum Energy," Phys. Rev. Lett. 112 (2014) 091304 arXiv:1309.6562 [hep-th]].

[10] C. de Rham, G. Gabadadze, L. Heisenberg and D. Pirtskhalava, "Cosmic Acceleration and the Helicity0 Graviton," Phys. Rev. D 83 (2011) 103516 [arXiv:1010.1780 [hep-th]].

[11] C. de Rham and L. Heisenberg, "Cosmology of the Galileon from Massive Gravity," Phys. Rev. D 84 (2011) 043503 arXiv:1106.3312 [hep-th]].

[12] G. Tasinato, "Cosmic Acceleration from Abelian Symmetry Breaking," JHEP 1404 (2014) 067 arXiv:1402.6450 [hep-th]].

[13] J. E. Kim and H. P. Nilles, "Dark energy from approximate $U(1)_{d e}$ symmetry," Phys. Lett. B 730 (2014) 53 [arXiv:1311.0012 [hep-ph]].

[14] A. S. Goldhaber and M. M. Nieto, "Photon and Graviton Mass Limits," Rev. Mod. Phys. 82 (2010) 939 arXiv:0809.1003 [hep-ph]].

[15] C. M. Will and K. Nordtvedt, Jr., "Conservation Laws and Preferred Frames in Relativistic Gravity. I. Preferred-Frame Theories and an Extended PPN Formalism," Astrophys. J. 177 (1972) 757.

[16] R. W. Hellings and K. Nordtvedt, "Vector-Metric Theory of Gravity," Phys. Rev. D 7 (1973) 3593.

[17] T. Jacobson and D. Mattingly, "Gravity with a dynamical preferred frame," Phys. Rev. D 64 (2001) 024028 gr-qc/0007031.

[18] J. D. Bekenstein, "Relativistic gravitation theory for the MOND paradigm," Phys. Rev. D 70 (2004) 083509 [Erratum-ibid. D 71 (2005) 069901] astro-ph/0403694].

[19] J. B. Jimenez, R. Durrer, L. Heisenberg and M. Thorsrud, "Stability of Horndeski vector-tensor interactions," JCAP 1310 (2013) 064 arXiv:1308.1867 [hep-th]].

[20] B. M. Gripaios, "Modified gravity via spontaneous symmetry breaking," JHEP 0410 (2004) 069 hep-th/0408127.

[21] L. Heisenberg, "Generalization of the Proca Action," arXiv:1402.7026 [hep-th].

[22] A. Nicolis, R. Rattazzi and E. Trincherini, "The Galileon as a local modification of gravity," Phys. Rev. D 79 (2009) 064036 [arXiv:0811.2197 [hep-th]].

[23] G. 't Hooft, "Naturalness, chiral symmetry, and spontaneous chiral symmetry breaking," NATO Adv. Study Inst. Ser. B Phys. 59 (1980) 135.

[24] M. A. Luty, M. Porrati and R. Rattazzi, "Strong interactions and stability in the DGP model," JHEP 0309 (2003) 029 hep-th/0303116.

[25] A. Nicolis and R. Rattazzi, "Classical and quantum consistency of the DGP model," JHEP 0406 (2004) 059 hep-th/0404159.

[26] C. Burrage, C. de Rham, D. Seery and A. J. Tolley, "Galileon inflation," JCAP 1101 (2011) 014 arXiv:1009.2497 [hep-th]].

[27] C. de Rham, "Galileons in the Sky," Comptes Rendus Physique 13 (2012) 666 arXiv:1204.5492 [astroph.CO]].

[28] A. I. Vainshtein, "To the problem of nonvanishing gravitation mass," Phys. Lett. B 39 (1972) 393.

[29] G. Tasinato, K. Koyama and N. Khosravi, "The role of vector fields in modified gravity scenarios," JCAP 1311 (2013) 037 arXiv:1307.0077 [hep-th]].

[30] S. Weinberg, "Adiabatic modes in cosmology," Phys. Rev. D 67 (2003) 123504 astro-ph/0302326.

[31] G. Tasinato, K. Koyama and G. Niz, "Vector instabilities and self-acceleration in the decoupling limit of massive gravity," Phys. Rev. D 87 (2013) 6, 064029 [arXiv:1210.3627 [hep-th]].

[32] K. Koyama, G. Niz and G. Tasinato, "The Self-Accelerating Universe with Vectors in Massive Gravity," JHEP 1112 (2011) 065 arXiv:1110.2618 [hep-th]].

[33] Work in preparation. 